\title{
Research on Video Monitoring System of Stage Surveillance Based on ONVIF Protocol
}

\author{
Zhen $\mathrm{Li}^{1,2,3}$, YuJie $\mathrm{An}^{1,2,3}$, YiChen Zhou ${ }^{1,2,3}$, WenJin $\mathrm{Wu}^{1,2,3}$ \\ 1School of Information and Engineering, Communication University of China \\ 2Key Laboratory of Acoustic Visual Technology and Intelligent Control System, Ministry of Culture \\ 3Beijing Key Laboratory of Modern Entertainment Technology \\ Beijing, China \\ E-mail: lizhen@cuc.edu.cn
}

\begin{abstract}
For the practical application requirements on stage surveillance and scheduling system, this paper has improved the existing video surveillance system with network control technology, streaming media technology, open-source VLC, ONVIF protocol. The improved video system can realize real-time monitoring of high-definition video, cradle head camera control, low time delay and can clearly display the performance of stage and emergency in time. Our research will provide technical basis for the networked stage surveillance system and has great value and prospect in application.
\end{abstract}

Keywords- Stage Surveillance; Video Monitoring; VLC; ONVIF Protocol.

\section{INTRODUCTION}

Stage supervision has been taken more and more seriously as one of the important guarantees of performance. The main surveillance and monitoring equipment is still given priority to analog equipment. Analog cameras capture images through radio-frequency signal after signal matrix in the end transmitting to monitors in monitoring platforms. In practical use, the video signal is always under different kinds of interference signal produced mainly by the running of stage machinery which will make it hard for the stage manager to understand the present situation of stage clearly. Nowadays because of the advantages in long distance transmission and anti-jamming, it will be more efficient and stable for video to transmit with video coding technology, streaming media technology and network protocol ${ }^{[1]}{ }^{[2]}$. Therefore, this paper proposed an improved video monitoring system based on ONVIF protocol which will realize the networked surveillance of stage for modern theatres.

\section{NETWORK VIDEO MONITORING SYSTEM OF STAGE}

The network video monitoring system of stage is made up of video camera terminal, network switches and streaming media server. As is shown in Fig.1.

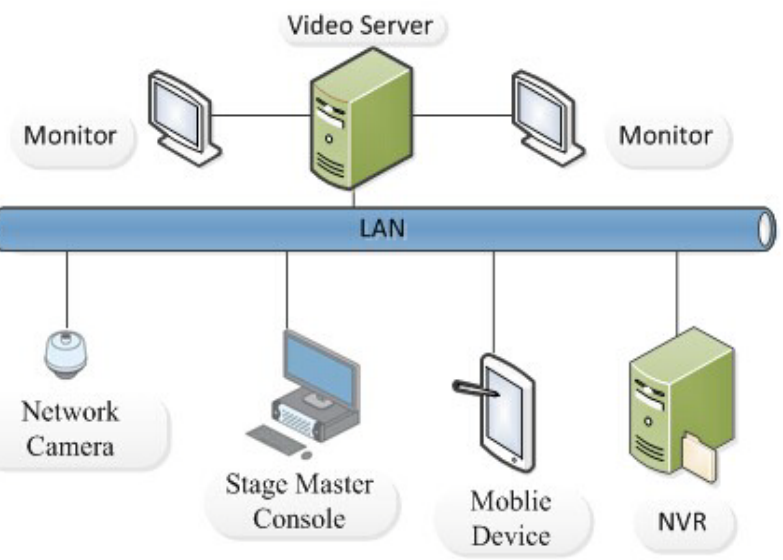

Fig.1. Network video monitoring system of stage

Front-end web camera will directly transmit video information to server. Then client software running on the server can monitor and control multiple points. Several network cameras and servers or other devices can communicate normally in the same local area network (LAN) by network switch. Due to interference between different kinds of equipment on stage, it is necessary to use shielded cables or optical fiber in data transmission and finish optical-electrical conversion at the termination point.

\section{ONVIF PROTOCOL AND VLC}

\section{A. ONVIF protocol}

ONVIF (Open Network Video Interface Forum), jointly initiated by three companies of SONY, AXIS and Bosch, is also an international forum name for network video product and technology development. So far there have been 
many product manufacturing enterprises of network video to join the group at home and abroad. ONVIF protocol is divided into different Profiles. Existing Profiles include Profile S, Profile C and Profile G. Each Profile has its special function. For example, Profile S mainly focuses on the function interfaces of video camera, including video and audio transmission of camera, video image set, multicast and cradle head camera control; Profile $G$ mainly involves receiving, storage and video footage management; Profile $\mathrm{C}$ is for network accessories management of security class including entrance guard, event alarming and so on. ONVIF protocol uses XML (Extensible Markup Language) as data description language, SOAP for message translation and WSDL (Web Services Description Language) to describe the services of protocol. The server and client in system parse the Request and Response to each other through the SOAP protocol. Specified in ONVIF audio and video streaming multimedia information between the server and client is controlled and transmitted through RTP and RTSP ${ }^{[3]}$. The regulatory structure of ONVIF is shown in Fig.2. ONVIF development process is shown in Fig.3.

\begin{tabular}{|c|c|c|c|}
\hline \multicolumn{2}{|c|}{ Media Plane } & \multicolumn{2}{|c|}{ Control Plane } \\
\hline \multicolumn{4}{|c|}{ Application/User Interface } \\
\hline \multicolumn{2}{|c|}{$\begin{array}{c}\text { Media Stream } \\
\text { Mediadata Stream }\end{array}$} & \multirow{2}{*}{$\begin{array}{c}\text { Device } \\
\text { Control } \\
\text { Media } \\
\text { Config }\end{array}$} & \multirow{2}{*}{$\begin{array}{l}\text { Meida } \\
\text { Control }\end{array}$} \\
\hline \multicolumn{2}{|c|}{ RTP/RTCP } & & \\
\hline \multicolumn{2}{|c|}{ RTSP } & SOAP & RTSP \\
\hline \multicolumn{2}{|r|}{ HTTP } & \multicolumn{2}{|c|}{ HTTP } \\
\hline \multirow{2}{*}{\multicolumn{2}{|c|}{ ILS }} & TLS & \\
\hline UDP & & \multicolumn{2}{|c|}{ TCP } \\
\hline \multicolumn{4}{|c|}{ IPV4/IPV6 } \\
\hline
\end{tabular}

Fig.2. ONVIF regulatory structure

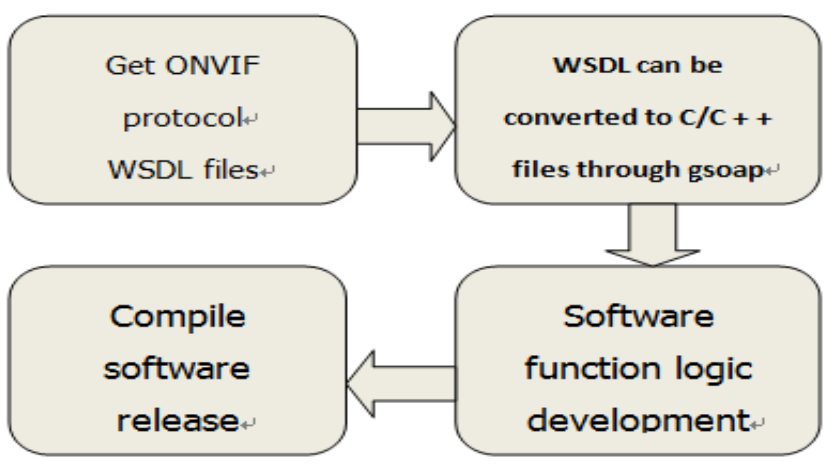

Fig.3. ONVIF development process

There are a variety of ways to develop ONVIF. The most common method is to use gsoap tool so that WSDL can be converted to $\mathrm{C} / \mathrm{C}++$ files. Then we can develop various functions.

\section{B. $V L C$}

VLC is an open source project of VideoLan, which can be used as multimedia player and streaming media server. It is compatible with multiple operating systems and can be used across platforms. VLC receives a variety of media inputs, supports multiple media formats and also can transcode different file formats. Player function will be classified into a number of modules by VLC. each module accomplishes certain function. The main modules include access, access_output, control, mux, demux, stream_output, video_output, interface and so on. Libvlc is the program entry of external reference, which is responsible for calling the function of VLC. As is shown in Fig.4.

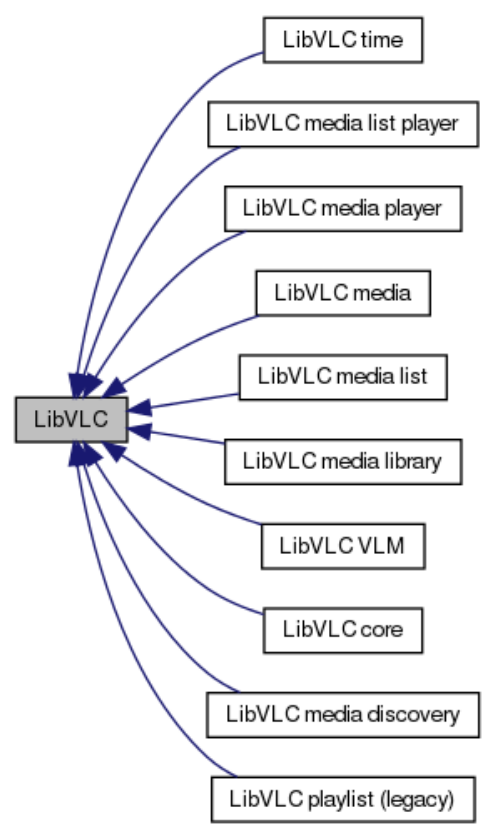

Fig.4. VLC Structure

\section{SOFTWARE FUNCTION DEVELOPMENT}

\section{A. Device discovery in ONVIF and cradle head control}

ONVIF protocol realizes device discovery mainly by sending broadcast messages to fixed port of fixed IP address in local area network (LAN) via UDP. This IP address is not necessary to be set with the same IP address of equipment. ONVIF protocol specifies that 239.255.255.250 is the fixed radio address. The port is 3702 and it cannot be modified or changed. By calling function soap_send__wsdd__Probe() we send broadcast messages to network and judge the returned int value for whether the messages have been sended successfully. If smoothly equipment in network will return part of basic information. At this time we can call soap_send__wsdd__ProbeMatch () to accept the returned device information, including: device type, device address, device management service, etc. 
Cradle head in ONVIF protocol is defined as an omni-directionally mobile equipment that has digital zoom capability (PTZ). ONVIF provides cradle head control with up, down, left, right, pan, tilt and zoom. If you want to control cradle head you have to get one or more control nodes of it. The node can be an entity of mechanism or a digital PTZ control equipment. After sending GetNodesRequest, we can read equipment response GetNodesResponse. If the node exits we can get the equipment PTZ node, on the contrary an error message.

Cradle head control class also provides SetPreset and GotoPreset that can let cradle head fast move to the preset.

\section{B. Video acquisition and network delay control of VLC}

Firstly, vlc player engine should be loaded by calling libvlc_new () to start vlc player. Then it is time to call libvc_media_new_location () to add media stream address into play item. After that, we can generate player via libvlc_media_player_new_from_media() and add the previous play item. Finally, performing libvlc_media_player_play () is able to achieve the acceptation and play of network media stream. At the end of the broadcast of media stream it needs to release instances of libvlc timely.

VLC has command control function which can be used to write control network command in cache through libvlc's instance libvlc_new. If network cache is set between $150-300 \mathrm{~ms}$, it delays in about 200ms and the picture is smooth without distortion.

const

char*

const

vlc_argv[]=\{"--network-caching $=200 "\}$;

static const int vlc_argv_nargs=sizeof (vlc_argv) / sizeof (vlc_argv[0]);

inst = libvlc_new (vlc_argv_nargs, vlc_argv);

$\mathrm{m} \quad=\quad$ libvlc_media_new_location(inst, urlName.toLatin1());

\section{SOFTWARE DEVELOPMENT PROCESS AND TESTING}

The software development process is shown in Fig.5. First of all, we should start the software and load the local libraries. Then it is time to turn on device search service, extract searched device's information and display which is shown in Fig.6. Connecting to device media stream address to get the media stream and show video pictures is the last step, as is shown in Fig.7. The device can be controlled by cradle head if necessary.

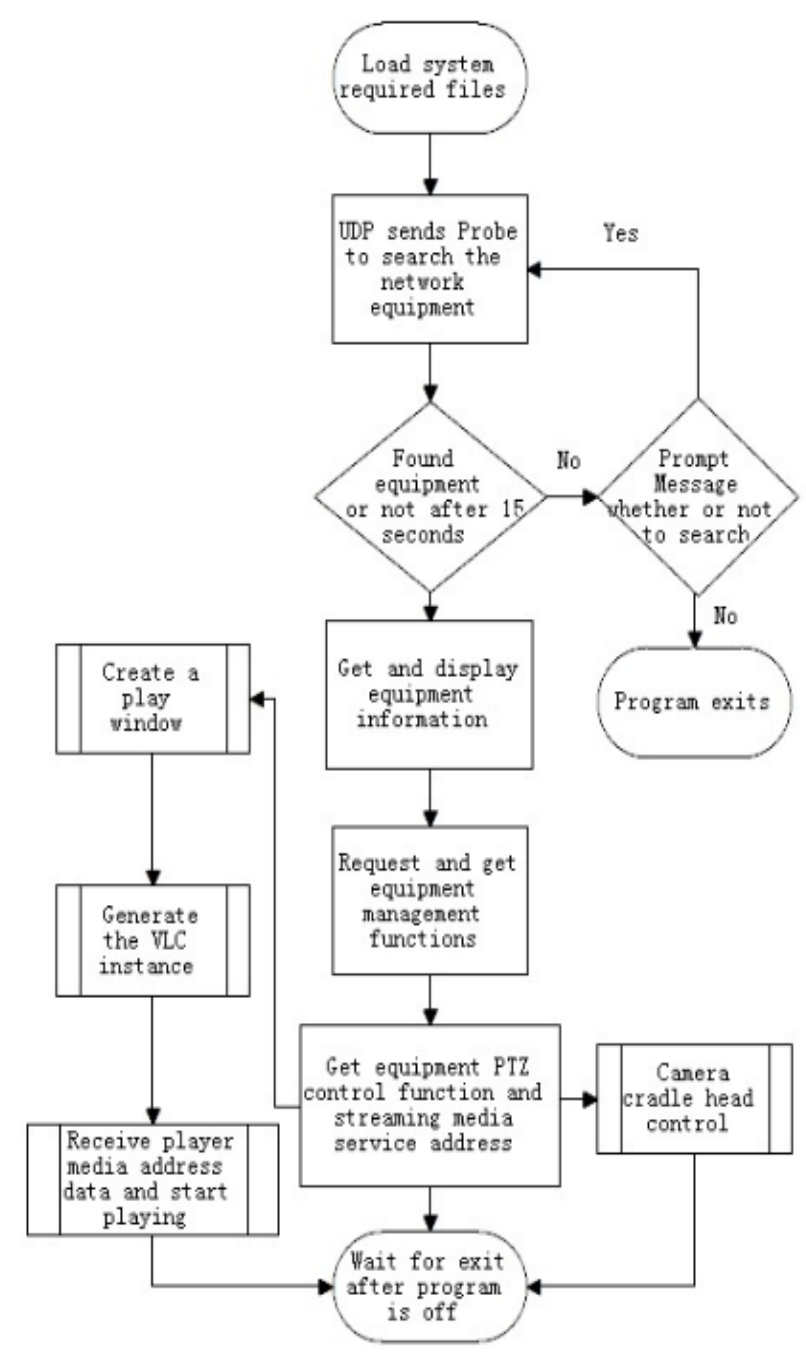

Fig.5.Software running processes based on ONVIF

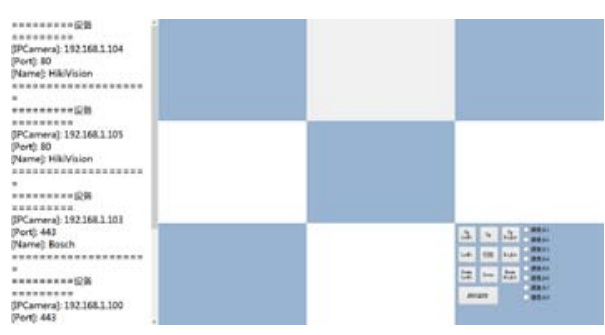

Fig.6. Equipment search function

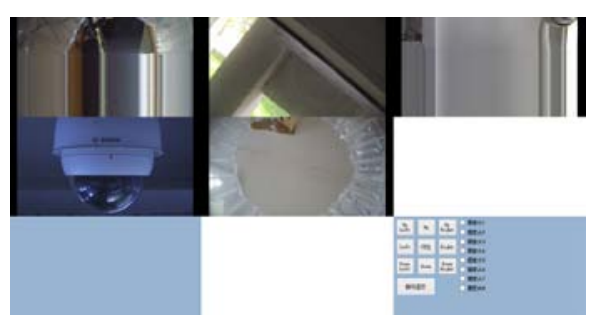

Fig.7. Monitoring interface

\section{CONCLUSION}

This paper has proposed an improved video monitoring system, which can realize data incoming of multipath video cameras and control of camera cradle head based on ONVIF protocol. The long-time running test proved that our system 
can provide high-definition real-time video for stage surveillance and accurate cradle head control by clicking the picture to the up and down through the mouse. The system will enrich the function of stage surveillance and scheduling system platform and provide clear, reliable and easy-to-use video interface for stage manager to grasp the real-time situation. Our research is of great value and prospect in application, which can provide technical basis for the networking of stage surveillance and scheduling system.

\section{ACKNOWLEDGEMENT}

This work was financially supported by the national science and technology planning project "Study and application demonstration on the key technology of the stage effect”(Item Number: 2012BAH38F00).

\section{REFERENCES}

[1] G.M. Zhong, "Stage supervisor system in Hangzhou Grand Theater,” Art Science \& Technology. 2004(2), pp.18-21.

[2] H. Wang, "Video surveillance system on Opera House,” Art Science \& Technology. 2008(2), pp.22-24.

[3] ONVIF Core Specification Version 2.4.1.[EB/OL] http://www.onvif.org/specs/core/ONVIF-Core-Specification-v241.pdf. 